\title{
Autophasing and lattice scaling in high energy linear accelerators
}

\author{
V.M. Tsakanov \\ Yerevan Physics Institute, Alikhanian Br. 2, 375036 Yerevan, Armenia
}

(Received 20 March 1998; published 31 August 1998)

\begin{abstract}
We have studied the autophasing conditions in long linear accelerators for a phase advance scaling along the machine instead of the usual beta scaling. A new lattice scaling provides the damping of the resonant enlargement of bunches in transverse wakefields with small required correlated energy spread in the bunch. In addition, such an approach allows one to keep the amplitude of coherent betatron oscillations small by a proper choice of the phase advance at the first cell. It is shown that the scaling of the phase advance per cell gives significant advantages with respect to singlebunch and multibunch instabilities. [S1098-4402(98)00013-5]
\end{abstract}

PACS numbers: 29.27.Bd, 29.17.+w, 41.75.-i, 41.85.-p

\section{INTRODUCTION}

The emittance preservation of the accelerated beams in future linear colliders is one of the basic problems which has to be solved to achieve design luminosity of the colliding beams. A list of sources causing effective emittance enlargement in linear colliders includes single particle (dispersion) and current dependent (wakefield) effects, which are the consequences of coherent betatron oscillations of the beam, imperfections of the machine components (misalignments of quadrupole and accelerating sections), and ground motion [1]. An important part of the beam dynamics issues in linear colliders is the technique of Balakin-Novokhatsky-Smirnov (BNS) damping [2], which provides the reduction of resonant enlargement of beams caused by wakefields. The autophasing conditions, known as one of the consequences of BNS damping in linear accelerators, provide that the transverse kicks to trailing particles caused by wakefields are, on average, compensated by the corresponding additional focusing on the quadrupoles of the lattice. This is accomplished by lowering the energy of the trailing particles relative to the head so that they are more strongly focused by the quadrupole lattice.

In general, for a complete suppression of short range wakefield and dispersive effects ("autophasing"), one has to scale the lattice parameters along the linear accelerator. The well-known scaling of the betatron wave number (beta function), with constant phase advance per cell, satisfies the autophasing conditions in each FODO cell $(\mathrm{F}=$ focusing quadrupole, $\mathrm{D}=$ defocusing quadrupole, $\mathrm{O}=$ drift space) if the beta function grows with the square root of the energy along the linear accelerator [3]. If multiple bunches are used in the linear collider to increase the luminosity, the long range wakefields become the source of the multibunch beam instability [4]. The autophasing conditions imply an increase in beta with the square root of energy, which in turn increases the amplitude of coherent betatron oscillations and induces the multibunch instability.
Another approach is to scale the phase advance per cell along the linear accelerator via the chromaticity of the focusing lattice keeping the cell arrangement unchanged $[5,6]$. Following this approach, in this paper we study the autophasing conditions when the beam performs coherent betatron oscillations down to the main linear accelerator. First, we define the basic scaling law of the focusing lattice as a function of the beam energy and apply it to the design of the linear optics of the linac. Then, we study the dependence of the singlebunch and multibunch instabilities of the beam on the focusing lattice parameters in the first order approximation in which the driving particles feel no wakefields. Finally, we analyze the autophasing conditions with scaled chromaticity along the linac and the corresponding reduction of the amplitude of coherent betatron oscillations in terms of the parameters of the focusing lattice.

\section{LATTICE SCALING}

The focusing cells along the linac are assumed to be a simple symmetric FODO lattice containing two accelerating sections. In thin lens approximation the analytical formulas for the periodic lattice functions are well known

$$
\begin{gathered}
\beta_{\text {max } \text { min }}=L_{c} \frac{1 \pm \sin \frac{\mu}{2}}{\sin \mu}, \quad \bar{\beta}=\frac{L_{c}}{\sin \mu}, \\
\xi=\frac{1}{4} K L_{q}\left(\beta_{\max }-\beta_{\min }\right)=\tan \frac{\mu}{2},
\end{gathered}
$$

where $\mu$ is the phase advance per cell, $L_{q}$ is the quadrupole length, $L_{c}$ is the cell length, $K$ is the focusing strength of the quadrupoles, $\beta$ is the usual beta function, $\bar{\beta}$ is an average beta function, and $\xi / \pi$ is the chromaticity of the FODO lattice. Note that the approximation of $\bar{\beta}$ is slightly different from the exact average beta, given by

$$
\bar{\beta}_{\text {exact }}=\frac{L_{c}}{2}\left(\cot \frac{\mu}{2}+\frac{2}{3} \tan \frac{\mu}{2}\right) .
$$

As the beam is accelerated, the normalized quadrupole gradient $K$ of a given magnetic field decreases inversely 
with the beam energy. If the cells are not scaled, the lattice is no longer symmetric and the beta function will increase linearly with energy while the phase advance per cell decreases. Since many of the tolerances depend upon the local value of the beta function, this is not a good choice. Another reason arises from the dependence of the dispersive and wakefield emittance dilutions on the scaling of the focusing lattice along the linac [7]. Although the normalized natural emittance of the beam is conserved, the rms emittance of the beam will increase due to the dispersive and wakefield effects, which are strongly dependent on the focusing lattice properties.

The following basic scaling of the phase advance per cell via the chromaticity of the focusing lattice will be used in further consideration

$$
\tan \frac{\mu_{n}}{2}=\tan \frac{\mu_{1}}{2} g_{n}^{\alpha},
$$

where $n$ is the cell number $g_{n}=\gamma_{0} / \gamma_{n}, \gamma_{0}$ is the initial energy, $\gamma_{n}=\gamma_{0}+(n-1) \Delta \gamma$ is the energy at the entrance in the $n$th cell, $\Delta \gamma$ is the energy gain per cell, $\mu_{n}$ is the phase advance in the $n$th cell, $\alpha$ is the scaling law parameter, and $\mu_{1}$ is the phase advance per first cell. The cell length is assumed to be constant along the linac.

The corresponding machine performance (quadrupoles strength) can be obtained via the transport matrix formalism.

The periodical solution in the first cell reads as

$$
\begin{gathered}
M_{11}+M_{22}=2 \sqrt{\frac{\gamma_{0}}{\gamma_{1}}} \cos \mu_{1}, \\
\beta_{0}=\beta_{1}=\sqrt{\frac{\gamma_{1}}{\gamma_{0}}} \frac{M_{12}}{\sin \mu_{1}}, \\
\alpha_{0}=\alpha_{1}=\sqrt{\frac{\gamma_{1}}{\gamma_{0}}} \frac{M_{11}-M_{22}}{2 \sin \mu_{1}} .
\end{gathered}
$$

The nonperiodical solution in the $n$th cell is

$$
\begin{gathered}
\sqrt{\beta_{n} \beta_{n-1}}\left(M_{11}+M_{22}\right)=\sqrt{\frac{\gamma_{n-1}}{\gamma_{n}}}\left[\left(\beta_{n}+\beta_{n-1}\right) \cos \mu_{n}+\left(\alpha_{n-1} \beta_{n}-\alpha_{n} \beta_{n-1}\right) \sin \mu_{n}\right], \\
\beta_{n}=\frac{\gamma_{n}}{\gamma_{n-1}}\left(M_{11}^{2} \beta_{n-1}-2 M_{12} M_{21} \alpha_{n-1}+M_{12}^{2} \gamma_{x n-1}\right), \\
\alpha_{n}=\frac{\gamma_{n}}{\gamma_{n-1}}\left[-M_{11} M_{21} \beta_{n-1}+\left(M_{12} M_{21}+M_{11} M_{22}\right) \alpha_{n-1}-M_{12} M_{22} \gamma_{x n-1}\right],
\end{gathered}
$$

where $\alpha_{n-1}, \beta_{n-1}, \gamma_{x n-1}$ and $\alpha_{n}, \beta_{n}, \gamma_{x n}$ are the Twiss parameters at the beginning and at the end of the $n$th cell, $M$ is the transport matrix of the $n$th cell, $\mu_{n}$ is the phase advance in the $n$th cell, and $\gamma_{n-1}, \gamma_{n}$ is the Lorenz factor of the design particle at the entrance and exit of the $n$th cell. This approach provides a full matching of the betatron function along the linac with the exception of the injection point where the incoming beam should be matched to the machine ellipse. Typical fully matched beta functions with cell chromaticity scaled as the square root of $\gamma_{0} / \gamma_{n}$ are shown in Fig. 1 for the parameters of the S-band linear collider [8] design. The phase advance per cell is slowly reduced from $120^{\circ}$ down to $60^{\circ}$ as the beam is accelerated from injection energy $3.15 \mathrm{GeV}$ up to $30 \mathrm{GeV}$ ( $\sim 150$ cells). The beta change at the low energy part is caused by the beam acceleration and the corresponding modification of the beta envelope (nonsymmetric lattice).

For comparison of the scaling of the phase advance with constant cell length and the usual scaling of the betatron function with constant phase advance per cell, we use two particular cases: the chromaticity scales inverse to the square root of energy and the beta scales as the squared root of energy.

The scaling of the beta function with energy $\left(\mu_{n}=\right.$ const) assumes that [3]

$$
\beta_{n \max }=\beta_{0 \max } g_{n}^{-1 / 2},
$$

$$
\begin{gathered}
\left(K_{1} L_{q}\right)_{n}=K_{0} L_{q 0} g_{n}^{1 / 2}, \quad L_{c n}=L_{c 0} g_{n}^{-1 / 2} \\
\bar{\beta}_{n}=\frac{L_{c 0}}{\sin \mu} g_{n}^{-1 / 2}
\end{gathered}
$$

while the scaling of the phase advance with energy $\left(L_{c n}=\right.$ const $)$ assumes that

$$
\begin{gathered}
\tan \frac{\mu_{n}}{2}=\tan \frac{\mu_{1}}{2} g_{n}^{1 / 2}, \\
\left(K_{1} L_{q}\right)_{n}=K_{0} L_{q 0} \frac{\sin \frac{\mu_{n}}{2}}{\sin \frac{\mu_{1}}{2}}, \\
\bar{\beta}_{n}=\frac{L_{c}}{2 \tan \frac{\mu_{1}}{2}}\left(g_{n}^{-1 / 2}+g_{n}^{1 / 2} \tan ^{2} \frac{\mu_{1}}{2}\right) .
\end{gathered}
$$

As will be shown in the following sections, these particular cases are the consequence of the autophasing conditions for a singlebunch acceleration in the linear accelerator.

\section{SINGLEBUNCH AND MULTIBUNCH INSTABILITIES}

We consider an ultrarelativistic bunched beam traversing a linac with a cylindrically symmetric accelerating structure and a FODO focusing lattice. As the beam travels through a large number of accelerating structures, strong wakefields are excited that affect the beam itself, leading to singlebunch and multibunch instabilities [9]. The 

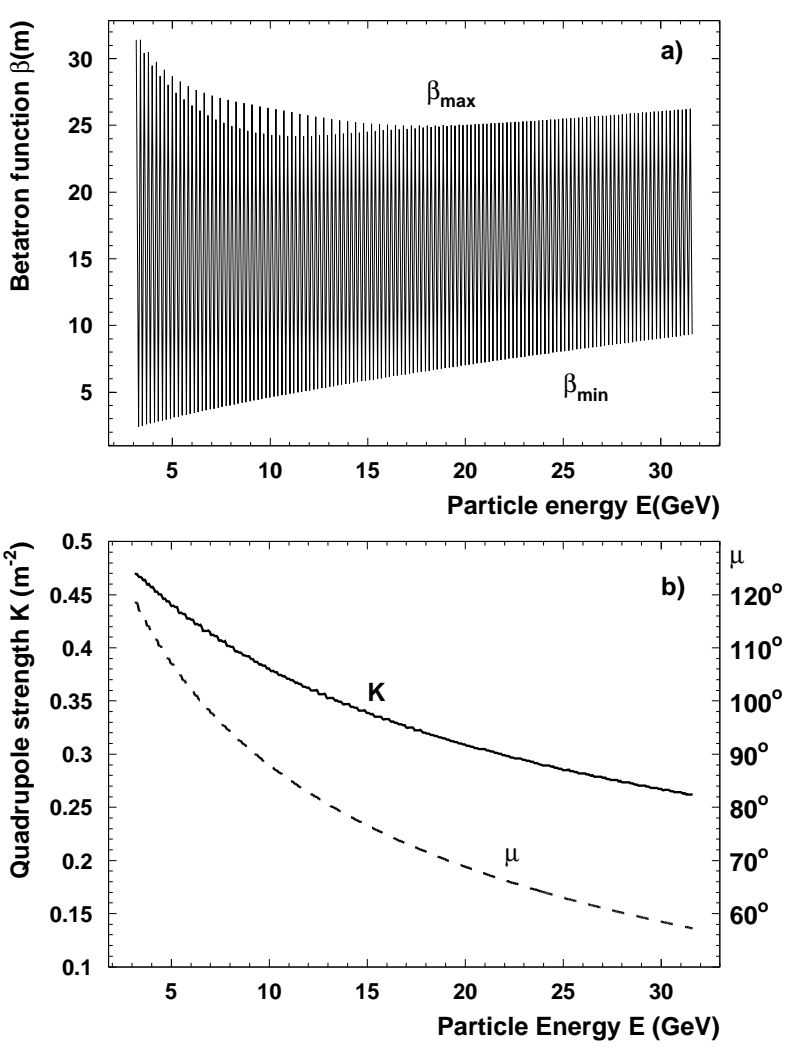

FIG. 1. The fully matched beta envelope along the main linac of the S-band linear collider with the chromaticity scaled as the square root of $\gamma_{0} / \gamma$ (a) and the corresponding variation of the phase advance (solid line) and quadrupoles strength (dashed line) along the main linac (b).

dominant effects in linear accelerators are due to the magnetic focusing fields and the transverse dipole wakefields. The transverse equation of motion for particles under the influence of transverse wakefields can be written as

$$
\begin{array}{r}
\frac{d}{d z}\left[\gamma(z, s) \frac{d}{d z} x(z, s)\right]+K_{x}(z, s) \gamma(z, s) x(z, s) \\
=N r_{e} \int_{-\infty}^{s} x\left(z, s^{\prime}\right) \rho\left(s^{\prime}\right) w_{x}\left(s-s^{\prime}\right) d s^{\prime},
\end{array}
$$

where $x$ is the transverse particle displacement, $z$ and $s$ are the longitudinal position in the accelerator and in the beam, $K_{x}$ is the normalized focusing strength, $\rho$ is the longitudinal distribution of the particle beam, $w_{x}$ is the dipole transverse wake potential of a point charge, $N$ is the number of particles, $r_{e}$ is the electron classical radius, and $\gamma$ is the Lorenz factor of the particle. The acceleration is approximately linear, $\gamma(z)=\gamma_{0}+G z$, where $G$ is the accelerating gradient.

The dynamics of a single bunch and of a train of bunches are different essentially for two reasons: the shape of the wake (short and long range wakefields) and the longitudinal distribution of the beam (Gaussian and the train of pointlike bunches). The dynamics of these processes have been studied in numerous Refs. [24,9-14]. We will consider the first order solution of the equation of motion which assumes that the driving particles (bunches) do not feel transverse wakefields and undergo free betatron oscillations with nonzero initial beam offset.

Let us start with the single bunch beam breakup in a linac. The simplest model that still contains the essential of the situation is a two particle model for the bunch [10]: the beam is modeled by two macroparticles with the charge $Q / 2$ that are separated by the longitudinal distance $\Delta s=2 \sigma_{s}$, where $\sigma_{s}$ is the rms length of the bunch. The head particle one feels no transverse wakefield and thus undergoes free betatron oscillations with the initial conditions $x_{0}, x_{0}^{\prime}$. The tail particle two experiences the dipole transverse wakefields $w_{1}$ due to the off-axis motion of the leading particle. Neglecting the energy spread, the particle two obeys the following equation of motion

$$
x_{2}^{\prime \prime}+\frac{\gamma^{\prime}}{\gamma} x_{2}^{\prime}+K x_{2}=\frac{N r_{e} w_{1}}{2 \gamma} x_{1}
$$

where prime denotes differentiation with respect to $z$. The solution of this inhomogeneous equation may be written in terms of the transport matrix $M\left(z_{0}, z\right)$. For the relative displacement $\Delta x=x_{2}-x_{1}$ of the tail particle with respect to the head particle we get

$$
\Delta x=\frac{1}{2} N r_{e} w_{1} \int_{0}^{z} M_{12}\left(z^{\prime}, z\right) \frac{x_{1}\left(z^{\prime}\right)}{\gamma\left(z^{\prime}\right)} d z^{\prime},
$$

with

$$
M_{12}\left(z^{\prime}, z\right)=\sqrt{\beta(z) \beta\left(z^{\prime}\right)} \sqrt{\frac{\gamma\left(z^{\prime}\right)}{\gamma(z)}} \sin \left[\phi(z)-\phi\left(z^{\prime}\right)\right],
$$

$$
x_{1}(z)=a \sqrt{\beta(z)} \sqrt{\frac{\gamma_{0}}{\gamma(z)}} \cos [\phi(z)-\theta] .
$$

Here $a, \theta$ define the initial amplitudes of free betatron oscillations and $\phi(z)$ is the betatron phase advance. Neglecting quickly varying terms, we find the rms emittance enlargement by averaging $\Delta x^{2}$ over an actual betatron phase

$$
\begin{aligned}
\Delta \varepsilon(z)= & \frac{\left\langle\Delta x^{2}\right\rangle}{\beta(z)} \\
= & \frac{a^{2}}{2}\left(\frac{N r_{e} E_{r} w_{1} L_{c 0}}{4 G}\right)^{2} \\
& \times \frac{\gamma_{0}}{\gamma}\left(\frac{G}{E_{0} L_{c 0}} \int_{0}^{z} g\left(z^{\prime}\right) \beta\left(z^{\prime}\right) d z^{\prime}\right)^{2},
\end{aligned}
$$

where $E_{0}$ is the injection energy and $E_{r}$ is the rest energy of the electrons.

The basic mechanism of the multibunch instability is essentially the same as that of the single bunch instability: As a bunch in the beam pulse is displaced from the axis of the axial-symmetric accelerating structures, transverse deflecting modes are excited in the structure that lead to a beam blowup. The current is now composed of a 
train of bunches with identical charges $Q$ spaced by the distance $s_{b}$, which is a multiple of the rf wavelength of the accelerating mode. The bunches are considered to be rigid macroparticles, like delta functions. The transverse dipole wakefield force experienced by the $n$th bunch, spaced at $s=n s_{b}$ from the first bunch, is

$$
F_{\perp n}(z)=e Q \sum_{k=0}^{n-1} w_{x}\left[(n-k) s_{b}\right] x\left(k s_{b}, z\right) .
$$

Using the perturbation method [9], the first order solution $x_{n}^{(1)}$ of $n$th pointlike bunch is calculated from the free betatron oscillations of $n-1$ previous bunches. Neglecting again the energy spread and keeping the driving term in the solution of the equation of motion, we obtain

$$
\begin{aligned}
x_{n}^{(1)}(z)= & \frac{1}{2} N r_{e} W_{\perp_{n}} a \sqrt{\beta(z)} \sin [\phi(z)-\theta] \\
& \times\left(\frac{\gamma_{0}}{\gamma(z)}\right)^{1 / 2} \frac{G}{E_{0} L_{c 0}} \int_{0}^{z} g\left(z^{\prime}\right) \beta\left(z^{\prime}\right) d z^{\prime},
\end{aligned}
$$

with

$$
W_{\perp n}=\sum_{k=0}^{n-1} w_{x}\left[(n-k) s_{b}\right] .
$$

Then the rms emittance enlargement of the first order solution averaged over the actual phase is

$$
\begin{aligned}
\Delta \varepsilon(z)= & \frac{\left\langle x_{n}^{2}\right\rangle-\left\langle x_{n}\right\rangle^{2}}{\beta(z)} \\
= & \frac{a^{2}}{2}\left(\frac{N r_{e} E_{r} \sigma_{w} L_{c 0}}{2 G}\right)^{2} \\
& \times \frac{\gamma_{0}}{\gamma}\left(\frac{G}{E_{0} L_{c 0}} \int_{0}^{z} g\left(z^{\prime}\right) \beta\left(z^{\prime}\right) d z^{\prime}\right)^{2},
\end{aligned}
$$

with

$$
\sigma_{w}^{2}=\frac{1}{N_{b}} \sum_{k=1}^{N_{b}} W_{\perp n}^{2}-\left(\frac{1}{N_{b}} \sum_{k=1}^{N_{b}} W_{\perp n}\right)^{2} .
$$

Here $N_{b}$ is the number of bunches in the bunch train. Thus the contribution of the focusing lattice to the driving term in the solution for both the singlebunch and the multibunch regimes is described by the dimensionless parameter

$$
\mathcal{F}=\frac{G}{E_{0} L_{c 0}} \int_{0}^{z} g\left(z^{\prime}\right) \beta\left(z^{\prime}\right) d z^{\prime}
$$

which is an integral over the accelerating sections and gives the dependence of the beam breakup on the focusing properties of the lattice.

In general, the dynamics of the process is more complicated $[9,14]$, but the effect of the focusing system is described by the parameter $\mathcal{F}$, which directly depends on the lattice scaling along the linac. Replacing the beta values in the accelerating sections with their average values and passing them to the integration over the energy range, we obtain

$$
\mathcal{F}(g, \alpha)=\frac{1}{L_{c 0}} \int_{g}^{1} g^{-1} \bar{\beta}(g) d g .
$$

If the chromaticity of cells is scaled along the linac we get

$$
\begin{aligned}
\mathcal{F}(g, \alpha)= & \left(2 \alpha \tan \frac{\mu_{1}}{2}\right)^{-1} \\
& \times\left[\left(g^{-\alpha}-1\right)-\tan ^{2} \frac{\mu_{1}}{2}\left(g^{\alpha}-1\right)\right] .
\end{aligned}
$$

In the case of the beta scaling along the linac we get

$$
\mathcal{F}(g, \alpha)=(\alpha \sin \mu)^{-1}\left(g^{-\alpha}-1\right) .
$$

Note that both scaling laws give an increased absolute emittance dilution with energy if the parameters are scaled with $\alpha>1 / 2$. However, the chromaticity scaling still has a degree of freedom in the phase advance per first cell, which may completely change the evolution of the beta function along the linac.

For comparison, we consider the relative enlargement of the beam emittance when the beta scales along the linac with the square root of energy and the chromaticity scales with the square root of $g=\gamma_{0} / \gamma$. Assuming the same arrangement and phase advance $\mu_{1}=\pi / 2$ in the first cell, we get the following relative emittance enlargement caused by the transverse wakefields for a large number of cells $(g \ll 1)$

$$
\begin{gathered}
\frac{\Delta \varepsilon}{\varepsilon} \sim A g^{-1}\left(1-2 g^{1 / 2}\right) \text { (beta scaled) } \\
\frac{\Delta \varepsilon}{\varepsilon} \sim \frac{1}{4} A g^{-1}(1-g)(\text { chrom. scaled }) .
\end{gathered}
$$

Note that even in this nonoptimal case, the emittance dilution due to the wakefields is reduced by a factor of $1 / 4$ when the chromaticity is scaled along the linac. In the case of constant beta scaling along the linac, we get the well-known logarithmic growth of the relative normalized emittance dilution with energy [11]

$$
\frac{\Delta \varepsilon}{\varepsilon} \sim A \ln ^{2} g
$$

In Fig. 2 we show the emittance enlargement (via the parameter $\mathcal{F}$ ) at the low energy part of the main linac for a different phase advance per first cell. The chromaticity scales as the square root of $\gamma_{0} / \gamma$. The emittance enlargement for beta scaled as the square root of energy (dashed line) and for a constant beta lattice (dotted line) are also shown for comparison. For the investigated low energy part of the linac, the optimal phase advance of the first cell is about $130^{\circ}$. It decreases slowly to $40^{\circ}$ as the beam is accelerated up to the energy $\gamma=30 \gamma_{0}$. Note that in this case the emittance enlargement is close to that of the constant beta lattice, which we consider to be optimal with respect to the transverse wakefield effects. 


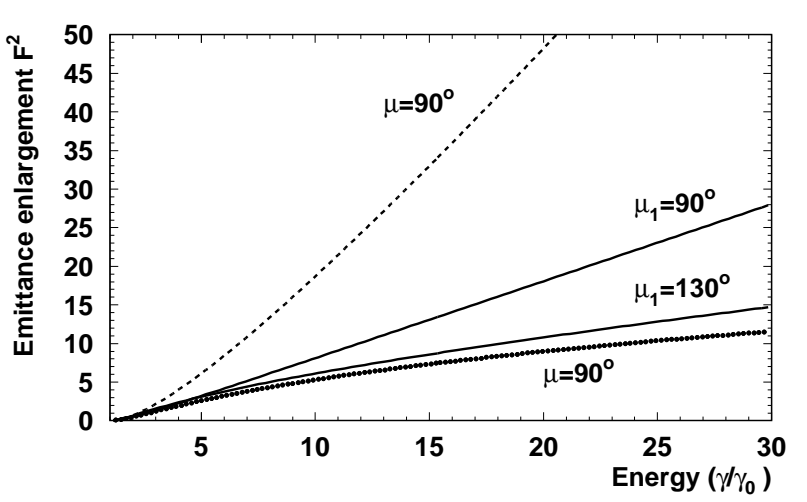

FIG. 2. The relative emittance enlargement factor $\mathcal{F}^{2}$ for different types of the lattice scaling along the linac. Shown are constant beta (dotted line), beta scaled as the square root of energy (dashed line) for a phase advance per cell $\mu=90^{\circ}$, and the chromaticity scaled as the square root of $\gamma_{0} / \gamma$ for a phase advance per first cell $\mu=90^{\circ}$ and $\mu=130^{\circ}$ (solid lines).

\section{BNS DAMPING}

Now we include the deviation of the correlated energy of the tail particle with respect to the head particle and look for the autophasing conditions when the chromaticity is scaled along the linac. We still use a two-particle model of the bunch and assume that the injected bunch has no initial correlated energy spread and the linac is fully filled by accelerating sections. As a short bunch travels through the accelerator, it gains energy from the externally driven accelerating mode and loses energy to the longitudinal wakefields. One can estimate the net relative correlated energy spread $\delta_{\text {cor }}$ of particle in the bunch along the linac as

$$
\delta_{\mathrm{cor}}(s, z)=\frac{\Delta E(s, z)}{E(z)}=\delta_{c}(s)[1-g(z)],
$$

where $\delta_{c}(s)=U(s) / E_{a}, U(s)$ is the net energy deviation of particles with the longitudinal position $s$ in the bunch with respect to the design energy $E(z)$ after the bunch has passed one accelerating section, and $E_{a}$ is the energy gain per accelerating section. Note that if the bunch has an initial relative correlated energy spread $\delta_{0}$, its contribution to the actual relative energy spread will decrease with energy

$$
\delta_{\text {cor }}(s, z)=\delta_{c}(s)[1-g(z)]+\delta_{0} g(z) .
$$

In the two-particle model, where the head particle has design energy $E(z)$ and feels no transverse wake force, and the trailing particle has the energy $E(z)\left(1+\delta_{\text {cor }}\right)$, the relative transverse displacement $\Delta x=x_{2}-x_{1}$ of the tail particle with respect to the head will obey the equation of motion

$$
\begin{aligned}
\Delta x^{\prime \prime}+\frac{\gamma^{\prime}}{\gamma} \Delta x^{\prime}+K(1 & \left.-\delta_{\mathrm{cor}}\right) \Delta x \\
& =\delta_{\mathrm{cor}} K x_{1}+C_{w} \frac{\gamma_{0}}{\gamma} x_{1},
\end{aligned}
$$

with $C_{w}=N r_{e} w_{1} / 2 \gamma_{0}$ for $|\Delta E / E|$ small. To calculate the BNS damping condition, we suppose free betatron oscillations of the head particle and use the $M_{12}$ matrix element to solve the equation. Since the autophasing cancellation is quasilocal, one can neglect the chromatic phase advance, which becomes significant only after a large number of cells. Thus we find the following expression for the relative transverse displacement after $N$ cells:

$$
\Delta x=a\left[g(z) \beta(z) \beta_{0}\right] \sum_{n=1}^{N}\left[\frac{\delta_{n}}{2} K_{n} L_{q}\left(\beta_{\max }-\beta_{\min }\right)+C_{w} L_{\text {acc }} g_{n} \bar{\beta}_{n}\right] \sin [\phi(z)-\theta]+\text { quickly varying terms } .
$$

Then, neglecting the chromatic phase advance and quickly varying terms, the autophasing condition in the $n$th cell will read as [3]

$$
\delta_{n}\left(K_{n} L_{q}\right)_{n}\left(\beta_{n \max }-\beta_{n \min }\right)+2 C_{w} L_{\mathrm{acc}} g_{n} \bar{\beta}_{n} \approx 0,
$$

where $L_{\text {acc }}$ is the accelerating section length. In terms of the phase advance per cell, we obtain

$$
4 \delta_{n} \tan \frac{\mu_{n}}{2} \approx-2 C_{w} L_{\mathrm{acc}} g_{n} \bar{\beta}_{n} .
$$

Taking into account the beta average and the correlated energy spread along the linac given by Eqs. (14) and (34), we obtain in the case of the chromaticity scaled with energy

$$
\delta_{c} g^{\alpha}+\left(\delta_{0}-\delta_{c}\right) g^{1+\alpha}=-\frac{1}{2} C_{w} L_{\mathrm{acc}} L_{c}\left(g^{1+\alpha}+g^{1-\alpha} \cot ^{2} \frac{\mu_{1}}{2}\right) .
$$

Thus the autophasing condition is satisfied for a chromaticity that scales as the square root of $g=\gamma_{0} / \gamma$, the additional 
initial correlated energy spread $\delta_{0}$, and the correlated energy spread per cell $\delta_{c}$ given by

$$
\begin{gathered}
\delta_{0}=-\frac{C_{w} L_{\mathrm{acc}} L_{c}}{4 \sin ^{2} \frac{\mu_{1}}{2}}, \\
\delta_{c}=-\frac{C_{w} L_{\mathrm{acc}} L_{c}}{4 \tan ^{2} \frac{\mu_{1}}{2}} .
\end{gathered}
$$

For comparison we cite also the autophasing solution for beta scaled as the squared root of energy [3]

$$
\delta_{0}=\delta_{c}=-\frac{C_{w} L_{\mathrm{acc} 0} L_{c 0}}{4 \sin ^{2} \frac{\mu}{2}} .
$$

Figure 3 shows the required correlated energy spread and the reduction of the multibunch wakefield emittance enlargement (via the parameter $\mathcal{F}$ ) as a function of the phase advance per first cell when the chromaticity is scaled as the square root of $\gamma_{0} / \gamma$ and the autophasing conditions are satisfied. Thus, even for a strong transverse short range wakefield, which is generally the case for linear accelerators with high operation frequency, the autophasing condition may be satisfied with relatively small correlated energy spread, while the amplitude of coherent betatron oscillations is kept small with the proper choice of the phase advance at the first cell $\mu_{1} \approx 2 \pi / 3$.

Note that with the use of the exact formula for the beta average (3), the reduction of the initial correlated energy spread is predicted as

$$
\delta_{0}=-\frac{1}{4} C_{w} L_{\mathrm{acc}} L_{c}\left(\cot ^{2} \frac{\mu_{1}}{2}+\frac{2}{3}\right),
$$

keeping the same requirement for a correlated energy spread induced in a cell. The reduction is of the order of $15 \%-25 \%$ when the phase advance for the first cell lies in the range of $90^{\circ}-130^{\circ}$ and can be elucidated by the particle tracking simulation only.

\section{SUMMARY}

We have obtained the autophasing conditions based on the matching of the dispersive term with the wakefield term to cancel the single bunch instability locally, when the beam performs coherent betatron oscillations down to the main linac. It was shown that the chromaticity scaling with the square root of $\gamma_{0} / \gamma$ allows one to reduce the required correlated energy spread. With the proper choice of the phase advance at the first cell, this technique allows one to keep small the amplitude of coherent betatron oscillations down to the linac and to reduce simultaneously the multibunch wakefield effects. In addition, the discussed approach provides beta matching along the linac by means of a slow variation of the phase advance per cell. The basic feature is that, with the proper choice of the phase advance per first cell, one can increase the dispersive term while keeping small the average amplitude of free betatron oscillations down to the main linac. In conclusion, we have to note that the choice of the optimal scaling law

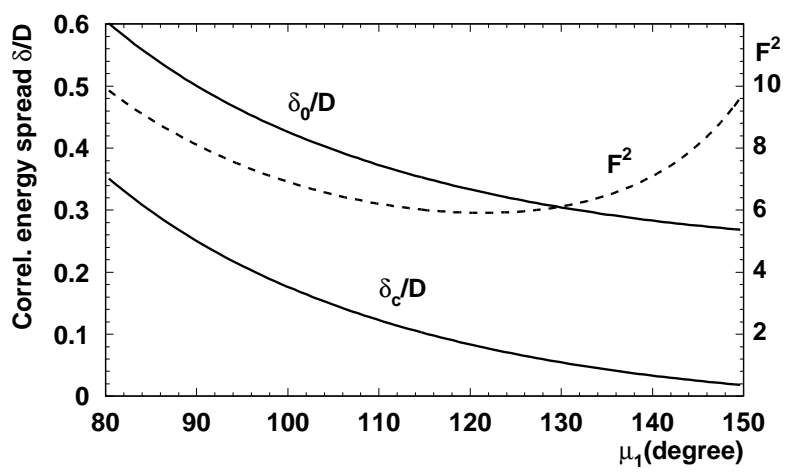

FIG. 3. An autophasing required $\operatorname{BNS}\left(\delta_{c} / D\right)$ and initial $\left(\delta_{0} / D\right)$ correlated energy spreads (solid lines) versus the phase advance at the first cell $\mu_{1}\left(D=C_{w} L_{\text {acc }} L_{c}\right)$. The dashed line shows the emittance enlargement factor $\left(\mathcal{F}^{2}\right)$ for a beam accelerated up to energy $\gamma=10 \gamma_{0}$. The chromaticity is scaled as the square root of $\gamma_{0} / \gamma$.

along the linac to preserve the beam emittance depends on many effects (misalignments, ground motion, etc.) as well as on particular peculiarities of linear collider projects.

\section{ACKNOWLEDGMENTS}

The author thanks R. Brinkmann and R. Wanzenberg for helpful discussions. Special thank is to G. Soehngen for permanent support of the work.

[1] J. T. Seeman, Report No. SLAC-PUB-4886, 1989.

[2] V. Balakin, A. Novokhatsky, and V. Smirnov, in Proceedings of the 12th International Conference on High Energy Accelerators (Fermilab, Batavia, IL, 1983), p. 119.

[3] T. O. Raubenheimer, Report No. SLAC-387, 1991.

[4] A. Chao, B. Richter, and C. Yao, Nucl. Instrum. Methods 178, 1 (1980).

[5] V.M. Tsakanov, LC92, ECFA Workshop on $e^{+} e^{-}$Linear Colliders, Garmisch-Partenkirchen, Germany (MaxPlanck Institut fur Physik, Munchen, 1993).

[6] V.M. Tsakanov, Report No. DESY M 96-02, 1996.

[7] G. Guignard, in Proceedings of the 1993 Particle Accelerator Conference, Washington, DC (APS, New York, 1993), p. 3600.

[8] Th. Weiland, in Proceedings of the 1990 Linear Accelerator Conference, Albuquerque, New Mexico (Los Alamos National Laboratory, Albuquerque, 1991), p. 792.

[9] A. W. Chao, Physics of Collective Beam Instabilities in High Energy Accelerators (Wiley, New York, 1993).

[10] P. Wilson in Physics of High Energy Particle Accelerators, edited by R. A. Carrigan, F. R. Huson, and M. Month, AIP Conf. Proc. No. 87 (AIP, New York, 1982), Sec. 11.1.

[11] K. Bane, Report No. SLAC-PUB-4169, 1986.

[12] K. Yokoya, Report No. DESY 86-084, 1986.

[13] H. Henke and W. Schnell, Report No. CERN-LEP-RF 86$18,1986$.

[14] A. Mosnier, in Proceedings of the 1993 CAS CERN Accelerator School on Advanced Accelerator Physics, Rhodes, Greece (CERN, Geneva, 1995), p. 459. 